\title{
EDITORIAL
}

\section{The complaints culture}

(C) The Author(s), under exclusive licence to The Royal College of Ophthalmologists 2021

Eye (2021) 35:3181-3182; https://doi.org/10.1038/s41433-021-01667-y

We love to complain; about breakfast, about the traffic and, notoriously, about the weather. However, when the complaint is about our professional work it ceases to be a social conversation and becomes a serious worry.

NHS managers actively encourage complaints. They reasonably believe it to be a form of feedback allowing them to improve services. Unfortunately when their efforts start to produce worrying statistics they develop parallel systems such as "PALS" (Patient Advisory and Liaison Services) to try to resolve matters before they are classified as "complaints". Having patient advocates clearly empowers patients faced with a system that many of them have difficulties navigating. What is not universally recognised is the complex effect on the clinician resulting from a complaint or a PALS investigation.

When a clinician receives a complaint, there are a number of reactions. Denial is common: "I had to wait an hour after my appointment time" is deemed trivial, even vexatious, but for a single mother with tenuous child-care arrangements, such a wait may cause real problems. Deflection is common: "The clinic was overbooked by the managers"-which of course may be true. If no obvious harm has been suffered, clinicians should look at how the situation which resulted in the complaint occurred, and what steps should be taken to prevent recurrence. However, there are some complaints that are obviously serious. These can include behaviour that is unacceptable for professionals either towards patients or with other hospital staff, or clinical incidents.

We live in a society where everyone expects a good result from medical treatment, and if this does not eventuate there is a tendency to assume that the unwanted outcome is due to an error and so there is some-one to blame. There are of course cases when there is a "breach of duty of care" and ensuing harm (thus "causation" for the lawyers), and such patients deserve compensation for loss and suffering. How these cases are dealt with locally (in terms of the clinician) is currently unknown, but the paper by Lim et al. [1] in this issue gives real insight into what is happening at a national level. They report 1032 complaints to the GMC about ophthalmologists over a 13 year period. Whilst the single largest outcome was closure at triage, $12 \%$ of cases were referred to their employers without investigations. A further $24 \%$ of cases were concluded following investigation and no sanctions were applied. Six percent resulted in advice, $3 \%$ in a warning, and 9 cases (1\%) in an undertaking. They note that the GMC records complaints against about $3 \%$ of ophthalmologists a year.

There is relatively little literature on patient complaints (nonmedical) in ophthalmology. There is a reported association with age [2] in that younger ophthalmologists in the USA were more likely to have a complaint lodged against them. They reported $42 \%$ of their study population of 1342 ophthalmologists associated with a major teaching hospital had at least one complaint in a 12-year period, which seems higher than the Lim study. An older English study [3] reported a complaint rate of 5 per 10,000 attendances at a single hospital over a 3-year period, but it is not possible to compare the two studies because of differences in methodology.

If one looks at reports from a state or provincial level, a report from Iran [4] over a 2-year period, $9.2 \%$ of all complaints related to ophthalmology, and of these $66 \%$ were considered to be medical negligence. A more recent paper from Canada [5] looked at complaints to the provincial regulator over a 5-year period. There were 372 complaints involving 211 ophthalmologists out of 448 practicing ophthalmologists and they note that almost $50 \%$ of ophthalmologists had a complaint to their regulator in this period, which is much higher than in the UK from Lim's data. The commonest complaints related to communication, billing practices, consent, procedural mishap, and documentation. The functioning of the NHS means that complaints about billing are unlikely to figure in UK statistics, and the GMC is said anecdotally not pursue complaints about doctors' fees in private practice unless there is a pattern of recurrent complaints. In this Canadian paper, 31\% of cases some action was taken, most commonly advice or a caution, but $1 \%$ were referred to the disciplinary committee.

Lim et al. correctly identify the serious stress doctors feel on being notified of a complaint to the GMC. Their livelihood and the time invested in training is potentially at risk, even with the knowledge that many complaints are closed in the early stages. Although help and support is available (as described by Lim et al.), doctors are not renowned for seeking out help. At Queen's Hospital Eye Department there has been a senior consultant with a "pastoral role" for over 20 years, and such an informal role should be more widespread.

If a complaint does raise the possibility of a breach of duty of care with harm, then a doctor in the NHS will be covered by Crown Indemnity absent the extraordinary situation of alleged criminal negligence (broadly speaking intentional acts). NHS trusts will have a legal department and full cooperation must be given with the understanding that the objective is usually to achieve the cheapest resolution and not reputation protection. For doctors working in the independent sector, professional indemnity insurance is of course mandatory, and the insurer should be notified as quickly as possible. Specialist legal and expert advice is mandatory to avoid unnecessary litigation, costs, and stress for the doctor.

It is not likely that we will see fewer complaints in the near future. We need to recognise them as a part of medicine in the twenty-first century and remind ourselves that they represent an opportunity to learn, and that in most cases they do not represent a threat to our continuing to practice.

Charles Claoué ${ }^{1{ }^{\circledR}}$
${ }^{1}$ EYE-LAW CHAMBERS $^{\circledR}$, London, UK. ${ }^{\bowtie}$ email: charles@dbcg.co.uk

\section{REFERENCES}

1. Lim CS, De Silva I, Banerjee, S. A review of fitness to practise referrals and investigations of ophthalmologists by General Medical Council in the United Kingdom. Eye. 2021. https://doi.org/10.1038/s41433-021-01665-0. 
2. Fathy C, Pichert J, Domenico H, Kohanim S, Sternberg P, Cooper W. Association between ophthalmologist age and unsolicited patient complaints. JAMA Ophthalmol. 2017:136. https://doi.org/10.1001/jamaophthalmol.2017.5154.

3. Chavan R, Porter C, Sandramouli S. Formal complaints at an eye hospital: a three-year analysis. Clin Gov. 2007;12:85-92. https://doi.org/10.1108/ 14777270710741447.

4. Daneshparvar $H$, Javadian A. Malpractice complaints against ophthalmologists referred to the state of legal medicine organization in Iran. Iran J Ophthalmol. 2008;20:4-8.

5. Saha R, Kabanovski A, Klejman S, Margolin E, Buys YM. Patients' complaints involving ophthalmologists in the province of Ontario, Canada: a 5-year review. Can J Ophthalmol. 2020;55:22-26. https://doi.org/10.1016/j.jcjo.2019.08.007.

\section{COMPETING INTERESTS}

The author declares no competing interests.

\section{ADDITIONAL INFORMATION}

Correspondence and requests for materials should be addressed to C.C.

Reprints and permission information is available at http://www.nature.com/ reprints

Publisher's note Springer Nature remains neutral with regard to jurisdictional claims in published maps and institutional affiliations. 